\title{
A Wireless Sensor Network-Based Defence Model against Selective Forwarding Attack
}

\author{
https://doi.org/10.3991/ijoe.v14i05.8651 \\ Yun Ji \\ Chongqing College of Electronic Engineering, Chongqing, China \\ jy20023801@163.com
}

\begin{abstract}
Due to low communication costs and convenient deployment, wireless sensor network has been widely applied in various fields. However, it still has some problems in the defence against selective forwarding attacks. To address these problems, this paper proposes a model against selective forwarding attacks, which is built on the threshold secret sharing mechanism and adopts the individualized path routing protocol. Through simulation test, this paper studies the effects of attack intensity on the successful transmission rate and communication load under the same network deployment and communication topology but at different node densities and average neighbourhood degrees. The results show that this model can effectively defend against forwarding attacks, also saves communication resource, offering a technical reference for similar studies.
\end{abstract}

Keywords-wireless sensor, individualized path, selective forwarding attack, defence

\section{Introduction}

Wireless sensor network (WSN) [1-2] consists of a large number of sensor nodes with wireless communication and computing capabilities deployed in the work area in a self-organized manner [3-5]. Its endings can sense and examine the external world, which greatly expands human's ability to perceive the outside world. Therefore, it is widely applied in many fields, such as environmental protection, national defence and military affairs, medical and health services and earthquake relief work [6]. The composition of a WSN is shown below:

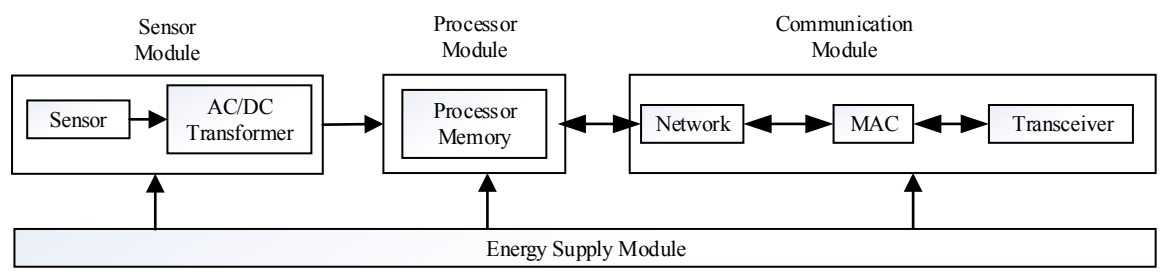

Fig. 1. Composition of wireless sensor network 
The first condition for the normal operation of a WSN is to ensure that the information collected by the sensing module is accurate and reliable when being transformed, analysed and transmitted [7-8]. Therefore, the security of network communications is crucial. However, due to its formation characteristics, WSN is vulnerable to various kinds of external and internal attacks such as eavesdropping and tampering during wireless communications [9]. Among them, selective forwarding attack is a kind of attack pattern by which the attacker located in the vicinity of the data transmission path obstructs, interferes with or blocks the normal delivery of information, causing key data to be lost [10-11]. This attack is very secretive and poses a serious threat to the security of an infinite sensor network [12]. In order to prevent selective forwarding attacks to the wireless sensor network, scholars at home and abroad have done a lot of research [13-14]. However, the current research methods still have many problems, like great communication overheads, occupancy of large storage space and not enough resistance to ensure accurate data transmission.

The SPREAD scheme based on the $(T, N)$ threshold secret sharing mechanism proposed by Lou [15] has certain resistance against the joint attack of malicious nodes. However, communication resources are seriously wasted as large amount of duplicate information is transmitted. This paper draws on the idea of secret sharing in the SPREAD scheme and proposes a selective forwarding defence model based on individualized path to handle selective forwarding attacks and save communication resources.

\section{Defence mechanism against selective forwarding attacks}

\subsection{Event division}

In the event region $\mathrm{i}$, the secret $D_{i}$ is split into $T_{i}$ shares and transmitted through $X_{i}$ paths; $j$ represents network node; $k_{j}$ is the only key to the node $\mathrm{j} . D_{i}$ is divided as shown in equation (1):

$$
f\left(k_{j}\right)=\left(d_{0}^{i}+d_{1}^{i} k_{j}+\ldots+d_{T_{i}-1}^{i} k_{j}^{T_{i}-1}\right) \bmod p j \in 1,2, \ldots, N
$$

$f\left(k_{j}\right)$ means the shares that node $\mathrm{j}$ needs to transmit, where $d_{t}^{i}$ is the split information of the secret $D_{i}$ with equal length $\left(\mathrm{t}=0,1, \ldots, T_{\mathrm{i}}-1\right)$. After the division, in the event region $i$, there will be $X_{i}$ paths for data share transmission. As long as the base station receives $T_{i}$ shares, it can obtain a complete secret $D_{i}$

The data share can be successfully delivered to the base station if and only if there is no packet loss caused by the malicious node along the path where the data share is delivered. Therefore, the probability $P_{i}$ of each data share of the secret $D_{i}$ in the event region $i$ being successfully transmitted to the base station is shown in equation (2):

$$
p_{i}=\left(1-\frac{n}{N-1} P_{d}\right)^{h_{i}} i \in 1,2, \ldots M
$$


From equation (2), we can see that the number of malicious nodes $n$, packet loss probability $P_{d}$ and node distance vector $h_{\mathrm{i}}$ are the three important factors that affect the successful reception of data. And the probability of successful reception decreases with the increase of these three factors.

The probability of the secret $D_{i}$ being successfully received is:

$$
P_{i}\left(k \geq T_{i}\right)=\sum_{k=T_{i}}^{X_{i}}\left(\begin{array}{c}
X_{i} \\
k
\end{array}\right) p_{i}^{k}\left(1-p_{i}\right)^{X_{i}-k}
$$

Due to the randomness of network deployment, the number of network nodes is different in different event regions and the shares $T_{i}$ of data divided are also different. As can be seen from the above formula, when the number of nodes and the probability of each data share being successfully transmitted are constant, the probability $P_{i}$ that the secret $D_{i}$ is successfully received increases with $T_{i}$ decreasing. But the smaller $T_{i}$ is, the higher the communication cost will be, so the value of $T_{i}$ should consider both security strength and communication cost.

In addition, the successful transmission rate of secrets can be expressed with the Beta function:

$$
P_{i}\left(k \geq T_{i}\right)=I_{p_{i}}\left(T_{i}, X_{i}-T_{i}+1\right)
$$

Suppose $P_{\exp }$ is the expected probability of the secret $D_{i}$ being successfully transmitted, then the reasonable value range for $T_{i}$ should be as shown in equation (5):

$$
P_{i}\left(k \geq T_{i}\right) \geq P_{\exp } \geq P_{i}\left(k \geq T_{i}+1\right)
$$

\section{$2.2 \quad$ Routing protocol}

In order to minimize the damages to the split data shares by malicious nodes, the greedy algorithm is used to find the neighbour that satisfies the conditions with the smallest distance vector from the neighbour table as the point for the next hop so as to make each data share forwarded by different intermediate nodes if possible. Suppose the execution node is $\mathrm{x}$, then the following algorithms are involved:

(1) Sending algorithm: find a neighbour that satisfies the following conditions, starting from the beginning of the neighbour table:

1) Hop count $\leq \mathrm{x}$.hop;

2) The event region is not $x$. eventid;

3) No share of x.eventid related event has been forwarded.

(2) Forwarding algorithm:

1) Find the sender node according to the sender id in the neighbour table;

2) Find, from the beginning of the neighbour table, a neighbour that satisfies the following conditions:

a Hop count < x.hop;

$\mathrm{b}$ The event region is not sender.eventid; 
c No share of sender.eventid related event has been forwarded.

(3). Monitoring algorithm: over-hearing

In the execution process of the sending algorithm and forwarding algorithm, the priority is as follows: the neighbour meeting the three conditions is given the first priority; otherwise the first neighbour is regarded as the routing node for the next hop.

\section{Performance analysis}

The hypothetic base station in this paper has sufficient storage space, strong computing capability and adequate power supply and is safe and reliable. The EIDs of all the nodes are controlled by the base station, and the attacker can capture any other node except in the base station. The performance of the above strategy is analysed from such aspects as storage load, communication load and security.

\subsection{Computation and storage loads}

The calculation of the secret split shares $T_{i}$ can be done at the base station or in the background. For a common sensor, regarding the secret $D_{i}$, only one $T_{i}$-1-order polynomial needs to be calculated, whose calculation amount depends on $T_{i}$, which is smaller than the number of nodes $\mathrm{X}_{i}$, so the calculation overhead is not large. In route selection, the neighbour table needs to be searched. The size of the neighbour table is determined by the density and communication distance of the network. Generally speaking, the neighbour degree $d$ is a few to a few dozens, so the routing algorithm is not computationally intensive. Regarding the storage space, the EIDs of the shares forwarded by routing protocols and nodes need to be stored, and the EID length is less than the node ID, so the storage space is not large.

\subsection{Communication load}

Node traffic is mainly determined by the data shares transmitted to the base station. Suppose the average distance vector is $h_{\mathrm{i}}$, and the data length is $\mathrm{L}$, then the most economical communication traffic $\mathrm{C}_{\mathrm{b}}$ is calculated as follows:

$$
C_{b}=\sum_{i=1}^{M} h_{i} L C_{b}=\sum_{i=1}^{M} h_{i} L
$$

Suppose the split isometric information $d_{t}^{i}$ of the secret $D i$ is of the same length $l_{i}$ as the node-calculated shares, then,

$\mathrm{L}=T_{i} l_{i} L$. When all shares are normally delivered to the base station, the maximum amount of information is

$$
C_{p}=\sum_{i=1}^{M} h_{i} X_{i} l_{i}
$$


Therefore, the total communication rate is

$$
R c=C_{p} / C_{b}=\sum_{i=1}^{M} h_{i} X_{i} / T_{i} / \sum_{i=1}^{M} h_{i}
$$

As can be seen, $R_{c}$ decreases with $T_{i}$ increasing, that is, the communication load decreases as the number of data shares decreases.

\subsection{Security}

In the path design, the dynamic routing affected by the order of communication between nodes is adopted, which makes it harder for the attackers to launch attacks. In addition, as each node shares a unique key with the base station, without knowing the key, even if the malicious node collects all the relevant data shares, it still cannot recover the secret $D_{i}$. At the same time, data shares will not be tampered with by a few malicious nodes. Even if the shares of the data forwarded are tampered with, the base station still can identify them through the redundant shares.

\section{$4 \quad$ Test and results}

\subsection{Test environment}

The 250 nodes are randomly and uniformly deployed on a $240 \times 240 \mathrm{~m}^{2}$ area, which is divided into 25 event regions. Let the base station ID be 0 and the location random.

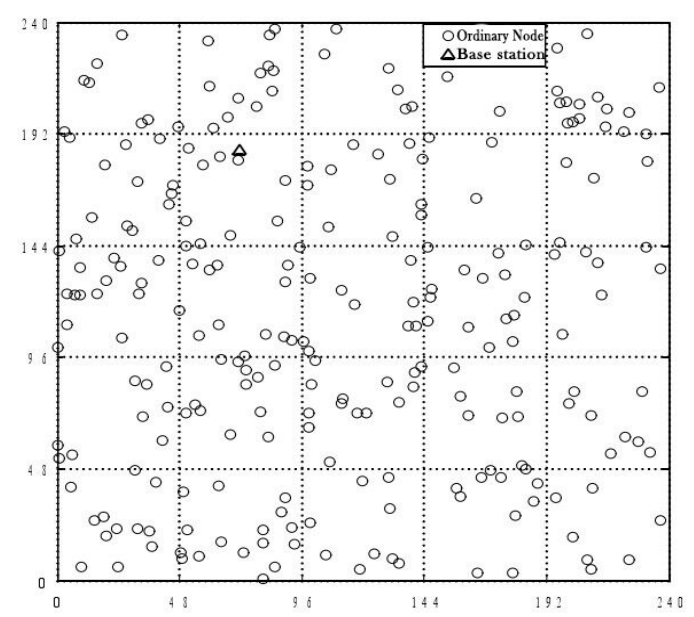

Fig. 2. Node deployment and event region division in the simulation experiment 
As the base station is trusted, malicious nodes are randomly assigned among the remaining nodes. In the simulation experiment, each time the locations of malicious nodes are different. Multiple rounds of experiments are performed with different parameters adjusted. In each round of experiment, the statistical average of 500 simulation results is used as the experimental result. In the simulation experiment, for simplicity, this paper does not consider the data tempering by malicious nodes, and corrects the communication load according to the successful transmission rate $\mathrm{P}$, which is $R_{c} / \mathrm{P}$.

\subsection{Test results}

Test 1: Under the same network deployment and communication topology, analyse the impacts of attack intensity on the successful transmission rate and communication load.

Given $\mathrm{d}=20, \mathrm{X}=10(\mathrm{X}=250, \mathrm{M}=25)$ and $\mathrm{P}_{\text {exp }}=0.85$, and the malicious packet loss rates are $0.1,0.2,0.5$ and 0.8 , respectively, 4 rounds of simulation tests are performed, with the results shown in Fig. 3 and Fig.4.

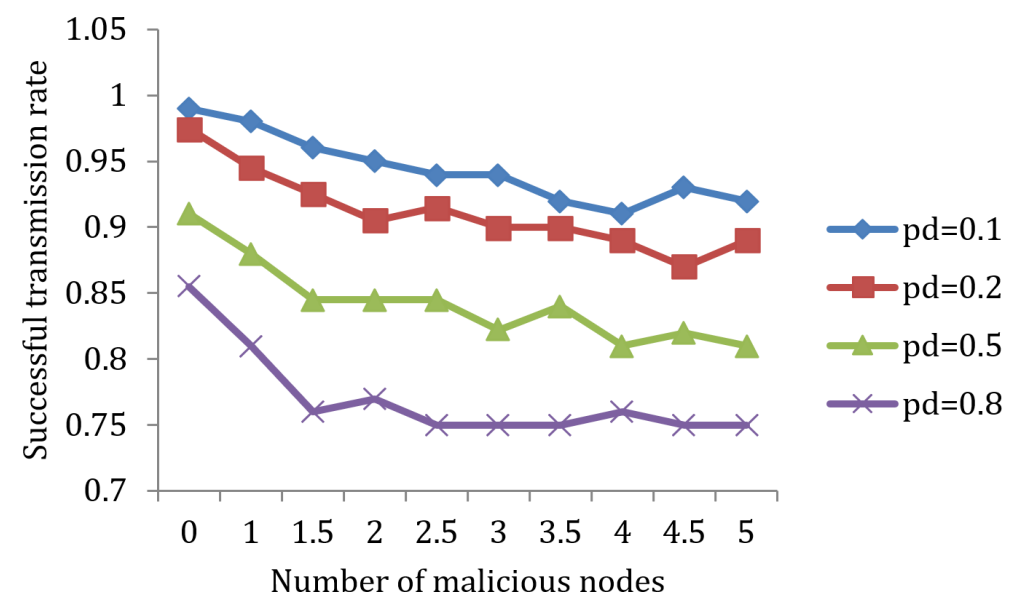

Fig. 3. Effects of different attack intensities on the successful transmission rate

As can be seen from the figure, attack intensity increases with the number of malicious nodes and the probability of packet loss increasing, but the decline of the successful transmission rate $P$ slows down. Therefore, the adjustment program for $T_{i}$ is effective. At the same time, experiments show that the successful transmission rate $\mathrm{P}$ decreases with the increase of the packet loss probability $P_{d}$. When $P_{d}=0.5,0.8$, the successful transmission rate is lower than expected. In addition, in order to maintain a relatively stable successful transmission rate, the communication cost increases as the number of malicious nodes increases. However, in our scheme, the relative communication load is less than 3 , even with the unsuccessful transmission excluded. 

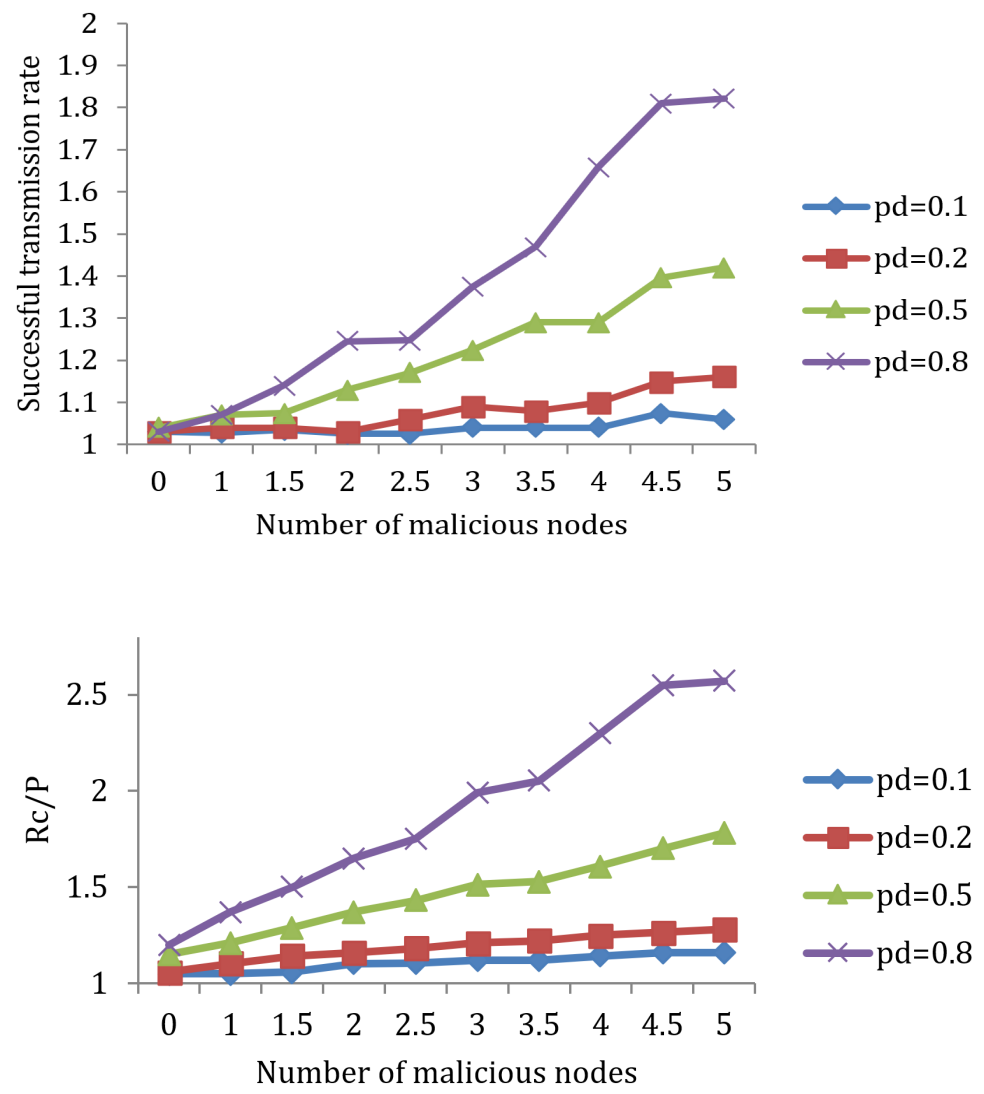

Fig. 4. Effects of different attack intensities on the communication load

Test 2: Effects of attack intensity on the successful transmission rate and communication load under different node densities.

In this experiment, $\mathrm{N}$ nodes in the network are adjusted and the average number of nodes $\mathrm{X}_{\mathrm{i}}$ is changed, in order to test the impacts of attack intensity on the successful transmission rate and communication load under different node densities. Let $\mathrm{N}$ be 200, 250 and 300, respectively, and then $X_{i}$ is 8,10 and 12. The malicious packet loss rate is fixed at 0.5, while $\mathrm{d}$ is maintained at 20. The results are shown in Fig.5 and Fig.6.

Experiments show that due to the $\left(\mathrm{T}_{\mathrm{i}}, \mathrm{X}_{\mathrm{i}}\right)$ threshold secret sharing mechanism adopted in this experiment, the sizes of $T_{i}$ and $X_{i}$ are correlated, which makes node density have little effect on the successful transmission rate. When the number of malicious nodes is constant, the smaller $\mathrm{N}$ is, i.e. the greater the relative attack intensity is, the more communication cost will be needed to maintain a stable successful transmission rate; in other words, the communication cost increases as the number of malicious nodes decreases. 


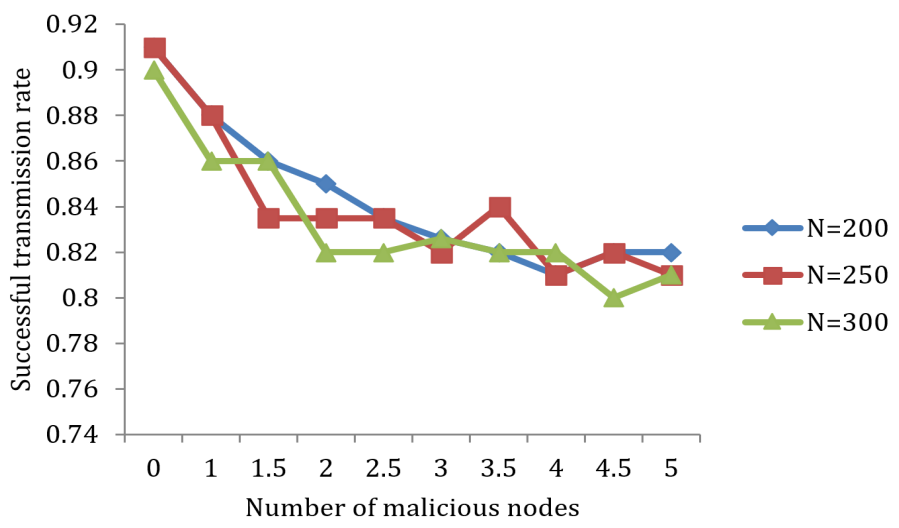

Fig. 5. Effects of attack intensity on the actual successful transmission rate under the condition of node density in different event regions
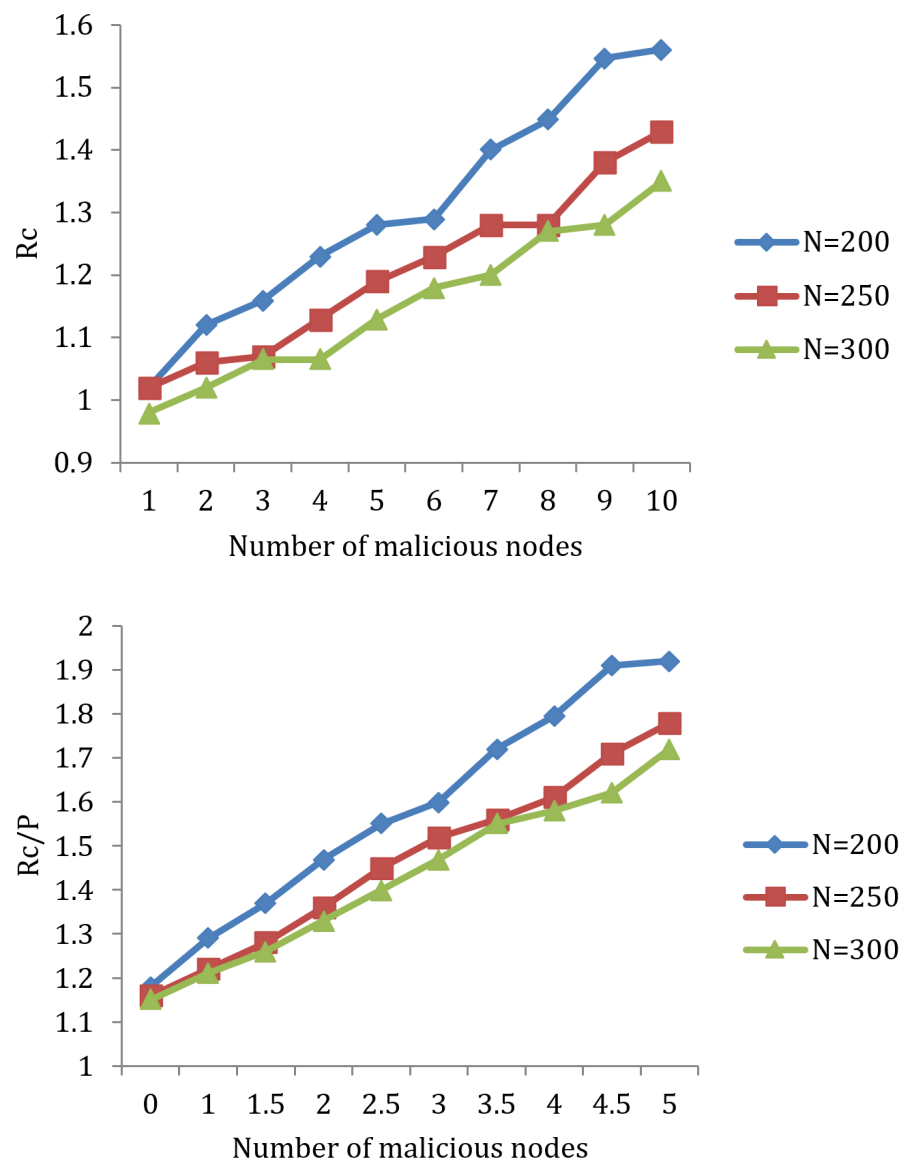

Fig. 6. Effects of attack intensity on the communication load under the condition of node density in different event regions 
Test 3: Effects of attack intensity on the successful transmission rate and communication load under different average neighbourhood degrees

The simulation is performed, given $\mathrm{X}=10, P_{\text {exp }}=0.85, P_{d}=0.5$ and $\mathrm{d}$ is $18,20,25$ and 28. The results are shown in Fig.8 and Fig.9.

The value of $T_{i}$ has considered the impact of communication hops, and the average neighbourhood degree $d$ mainly affects the number of communication hops, so it can be seen from the figure that the average neighbourhood degree $d$ has little effect on the successful transmission rate and communication load. When $d$ increases, the dispersion of the data share delivery path becomes better, which in turn makes the communication load smaller.

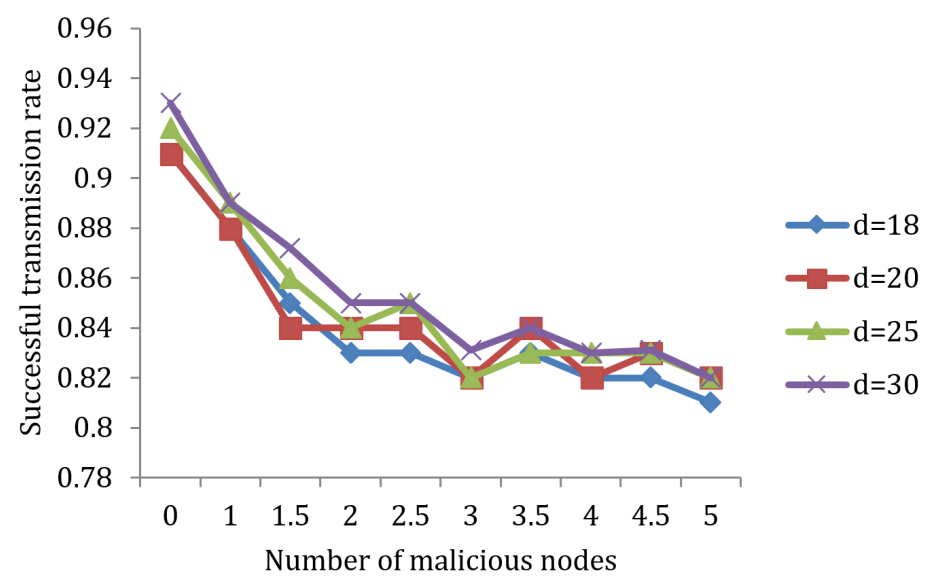

Fig. 7. Effects of attack intensity on the actual successful transmission rate under different node neighbour degrees

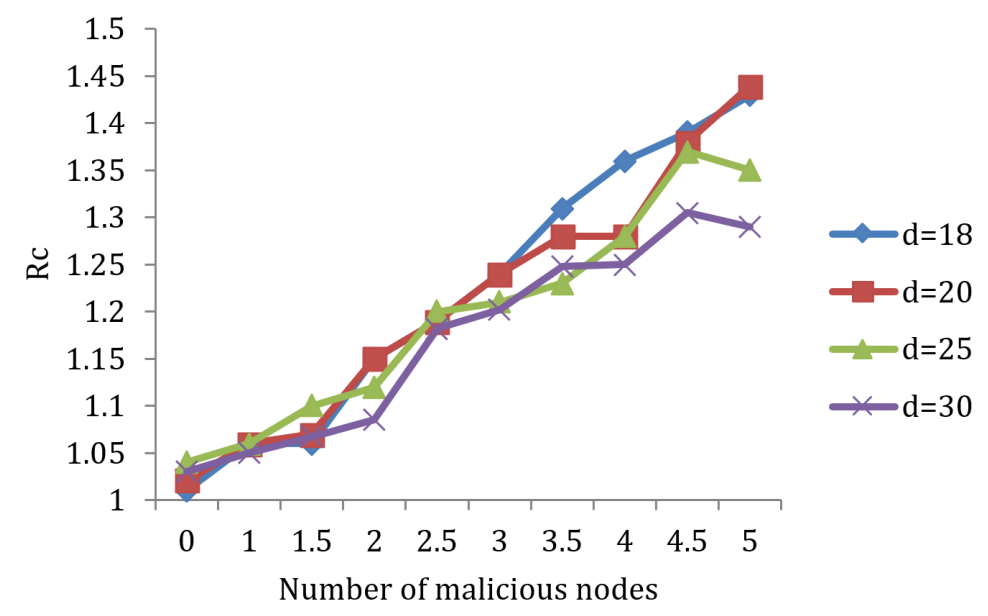




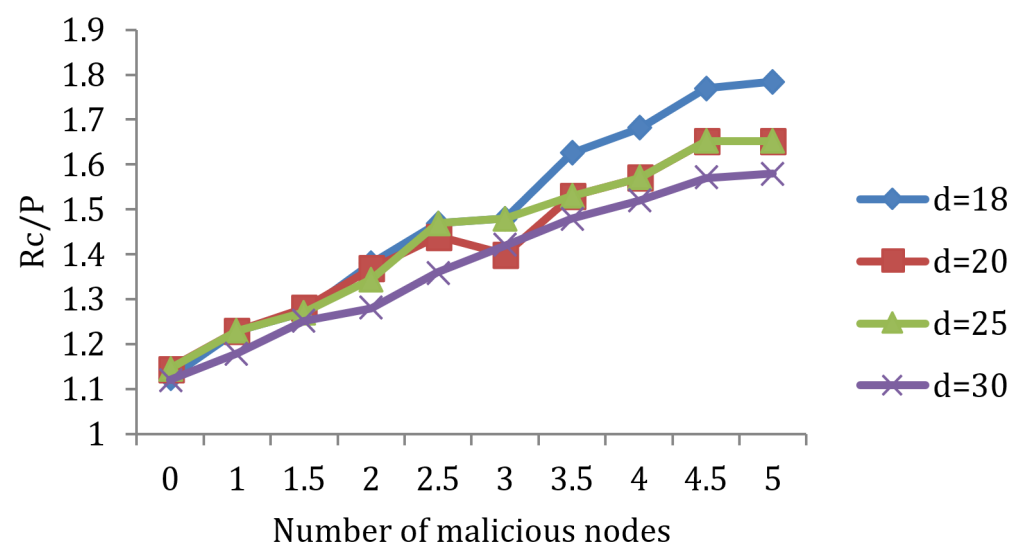

Fig. 8. Effects of attack intensity on the communication load under different node neighbour degrees

\section{Conclusions}

Based on the threshold secret sharing mechanism, this paper designs individualized path routing to defend against the selective forwarding attacks in the wireless sensor network. Through simulation test, it studies the effects of attack intensity on the successful transmission rate and communication load under the same network deployment and communication topology but at different node densities and average neighbourhood degrees. The following conclusions are obtained:

1. The successful transmission rate does not continuously drop with the number of malicious nodes and packet loss probability increasing; the communication load is still less than 3 even with the unsuccessful transmission excluded.

2. The node density has little impact on the successful transmission rate, and the communication cost increases with the number of nodes decreasing.

3. The average neighbourhood degree has little impact on either the successful transmission rate or the communication load. The communication load decreases with the average neighbourhood degree increasing.

\section{References}

[1] Akyildiz, I.F., Su, W., Sankarasubramaniam, Y. Cayirci, E. (2002). Wireless sensor networks: a survey. Computer Networks, 38(4), 393-422. https://doi.org/10.1016/S13891286(01)00302-4

[2] Li, H.M., Liu, H.H. (2017). The optimizated study on preparation process of nano tire pressure sensor used in auto, Academic Journal of Manufacturing Engineering, 15(1), 97 104. 
[3] Liu, M., Zheng, Y., Cao, J., Chen, G., Chen, L., Gong, H. (2007). Eadeeg: an energyaware data gathering protocol for wireless sensor networks. Journal of Software, 18(5), 1092-1109. https://doi.org/10.1360/jos181092

[4] Orito, E., Utani, A., Yamamoto, H. (2009). Pheromone-oriented routing protocol for load balancing of wireless sensor networks with multiple sinks (intelligent sensing and control). Journal of Japan Society for Fuzzy Theory \& Intelligent Informatics, 21(1), 56-68. https://doi.org/10.3156/jsoft.21.56

[5] Rawat, P., Singh, K.D., Chaouchi, H., Bonnin, J.M. (2014). Wireless sensor networks: a survey on recent developments and potential synergies. Journal of Supercomputing, 68(1), 1-48. https://doi.org/10.1007/s11227-013-1021-9

[6] Zhu, Y.H., Wu, W.D., Pan, J., Tang, Y.P. (2010). An energy-efficient data gathering algorithm to prolong lifetime of wireless sensor networks. Computer Communications, 33(5), 639-647. https://doi.org/10.1016/j.comcom.2009.11.008

[7] Sheu, J.P., Sahoo, P.K., Su, C.H., Hu, W.K. (2010). Efficient path planning and data gathering protocols for the wireless sensor network. Computer Communications, 33(3), 398408. https://doi.org/10.1016/j.comcom.2009.10.011

[8] Ramanan, K., Baburaj, E. (2010). Data gathering algorithms for wireless sensor networks: a survey. International Journal of Ad Hoc Sensor \& Ubiquitous Computing, 1(4), 393-422. https://doi.org/10.5121/ijasuc.2010.1410

[9] Bo, Y., and Bin,X. (2006). "Detcting selective forwarding attacks in wireless sensor networks" in Parallel and Distributed Processing Symposium, 2006. IPDPS 2006. $20^{\text {th }}$ International, 8, 2006.

[10] Xiao B., Yu, B., Gao, C.S. (2007). CHEMAS: Identify suspect nods in selective forwarding attacks, Journal of Parallel and Distributed Computing, 67(11), 1218-1230. https://doi.org/10.1016/j.jpdc.2007.04.014

[11] Kim C. Tao, W., Shin Kim, K. (2010). An empirical study of customers' perceptions of security and trust in e-payment systems. Electronic Commerce Research and Applications, (9), 84-95. https://doi.org/10.1016/j.elerap.2009.04.014

[12] Kurt G., Hacioglu, G. (2010). Ethics as a customer perceived value driver in the con-text of online retailing. African Journal of Business Management, 4(5), 672-677.

[13] Slovic, P., Finucane, M.L., Peters, E. (2004). Risk as Analysis and Risk as Feelings: Some Thoughts about Affect, Reason, Risk, and Rationality. Risk Analysis, 24(2), 311-322. https://doi.org/10.1111/j.0272-4332.2004.00433.x

[14] Sathye, M. (1999). Adoption of Internet banking by Australian consumers: an empirical investigation. International Journal of Bank Marketing, 17(8), 324-334. https://doi.org/10.1108/02652329910305689

[15] Lou, W., Liu, W., Fang Y. (2004). "SPREAD: Enhancing data confidentiality in mobile ad hoc networks, IEEE INFOCOM 2004, HongKong, China, March 2004.

\section{$7 \quad$ Author}

Ji Yun received B.Sc. degree and M.Sc. degree from Chongqing University and Chongqing Normal University. Now he is a lecturer in Chongqing College of Electronic Engineering. His main research interest is in electronic information engineering.

Article submitted 30 March 2018. Final acceptance 04 May 2018. Final version published as submitted by the author. 